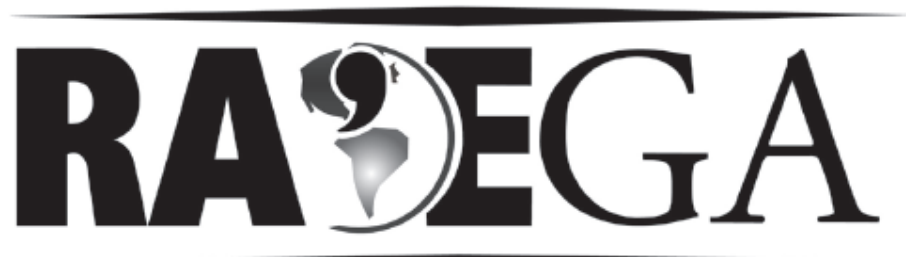

O ESPAÇO GEOGRÁFICO EM ANÁLISE

\title{
UTILIZAÇÃO DE SISTEMA DE INFORMAÇÃO GEOGRÁFICA NA ANÁLISE DA DISTRIBUIÇÃO DE CETÁCEOS NA COSTA PARANAENSE
}

\section{USE OF GEOGRAPHIC INFORMATION SYSTEM IN THE ANALYSIS OF DISTRIBUTION OF CETACEANS IN PARANA COAST}

\author{
Ana Carolina Vizintim Fernandes Barros ${ }^{1}$ \\ Mirian Vizintim Fernandes Barros ${ }^{2}$
}

\begin{abstract}
Resumo
A distribuição geográfica de cetáceos é importante para o seu monitoramento e gestão, no entanto este conhecimento enfrenta algumas dificuldades, tais como, a obtenção de dados sobre sua ocorrência, e a disponibilidade e acesso às informações. Estes dados são obtidos a partir de técnicas de avistagem, captura acidental, encalhe e tombamento. $O$ armazenamento, manipulação e analise destes dados podem ser realizados pelo Sistema de Informação Geográfica - SIG, que é uma importante ferramenta, pois, permite a manipulação de um grande volume de dados e sua atualização, e também possibilita a representação da distribuição. Este artigo apresenta um estudo realizado na costa do litoral paranaense a partir de dados de materiais tombados levantados do Museu de Historia Natural Capão da Imbuia e do Museu de Ciências Naturais da Universidade Federal do Paraná, que somaram 197 registros, com objetivo de elaborar um banco de dados sobre estas ocorrências e sua distribuição geográfica.
\end{abstract}

Palavras-chave: cetáceos; distribuição; material tombado; SIG e litoral paranaense.

\footnotetext{
1 Especialista em Gestão, Auditoria e Licenciamento Ambiental pela Universidade Norte do Paraná. Londrina, Paraná, Brasil (carol.vizintim@gmail.com).

2 Doutora em Geografia Física pela Universidade de São Paulo - USP; Professor Associado do Departamento de Geografia da Universidade Estadual de Londrina. Londrina, Paraná, Brasil (mirianvizintim@gmail.com).
} 


\section{Abstract}

The geographic distribution of cetaceans is important to its monitoring and management, however this knowledge confronts some difficulties, such as getting data on their occurrence, the availability and access to the information. These data are obtained from sighting techniques, bycatch, stranding and tipping. The storage, handling and analysis of these data can be performed by the Geographic Information System - GIS, which is an important tool because allows it the manipulation of large volumes of data and the updating, also allows the representation of the distribution. This paper presents a study on the coast of Paraná State with data collected from the Natural Museum of History Capon of Imbuia and the Natural Museum of Sciences, from the Federal University of Paraná, which summarized 197 records, with the goal of developing a database on these events and their geographical distribution.

Keywords: cetaceans; distribution; material tumbled; GIS and coast of Paraná.

\section{INTRODUÇÃO:}

Os cetáceos são mamíferos aquáticos de grande porte, podem ser marinhos ou de água doce, nas águas jurisdicionais brasileiras estão registradas 39 espécies no Plano de Ação para Mamíferos Aquáticos do Brasil (IBAMA, 2001). Segundo este Plano a Baleia Azul (Balaenoptera musculus) apresenta risco muito alto de extinção na natureza em futuro próximo; enquanto as Baleia-sei (Balaenoptera borealis), Baleia-fin (Balaenoptera physalus); Baleia Jubarte (Megaptera novaeangliae); Baleia-franca-do-sul (Eubalaena australis); Cachote (Physeter macrocephalus); Boto-amazônico (Inia geoffrensis); e Toninha (Pontoporia blainvillei) apresentam alto risco de extinção na natureza em médio prazo; a Baleia-minke-antártica (Balaenoptera bonaerensis) apresenta baixo risco e as demais os dados são insuficientes.

Conhecer as espécies que vivem nas regiões brasileiras é de grande importância para promover a conservação da biodiversidade da fauna marinha e para alcançar este objetivo são necessários estudos que considerem não apenas a biologia, mas também a influencia das ações antrópicas no seu habitat.

Os cetáceos conhecidos popularmente como botos, golfinhos e baleias, são divididos em duas subordens viventes: Mysticeti, baleias com barbatanas, e Odontoceti, os que possuem dentes como os golfinhos, toninhas e cachalotes. Os misticetos são exclusivamente marinhos e possuem um 
padrão de migração, e, são mais homogêneos em forma (REYNOLDS \& ROMMEL, 1999), enquanto que os odontocetos possuem grande diversidade de espécies e não apresentam padrões migratórios definidos (HETZEL \& LODI, 1993).

Entre as metodologias de estudo de ocorrência de cetáceos estão a avistagem, a captura acidental o encalhe (MORAES, 2005) esses métodos fornecem uma somatória de informações que permitem às instituições de pesquisa e de gestão elaborarem estratégias de proteção, conservação e manejo dessas espécies.

Grande parte dos registros de avistagem e encalhe são obtidos de forma oportunista, sendo restritos a determinadas regiões, a alguns pesquisadores e instituições. O método mais seguro para obter informações sobre a distribuição dos cetáceos é a observação direta; pratica naturalmente difícil, pois esses mamíferos passam somente em torno de $10 \%$ de seu tempo de vida na superfície (MORAES et al., 2004).

O encalhe de animais vivos ou mortos é atualmente segundo Medeiros (2006), a principal fonte de informação sobre a ocorrência, diversidade, biologia e ecologia da maioria das espécies de mamíferos marinhos. O estudo das carcaças de animais mortos não permite uma informação exata de seu habitat, pois muitas vezes o encalhe ocorre algum tempo após sua morte, e, a carcaça é transportada por correntes marinhas, por vezes longe do local de ocorrência.

O material tombado consiste em informações catalogadas sobre animais encalhados, e o arquivamento de parte ou totalidade do seu esqueleto ósseo. Este material arquivado em instituições especializadas é essencial para o desenvolvimento de estudos de cetáceos e sua biodiversidade por serem repositórios de espécimes-testemunho e por fornecer matéria-prima para a compilação de listas de espécies. São importantes também na compreensão dos padrões biogeográficos, na investigação das tendências evolutivas passadas e presentes, assim como na documentação das extinções e invasões (COSTA et al., 2005).

Embora o material tombado de cetáceos seja muito importante para 0 desenvolvimento de pesquisas e conhecimento desta espécie e sua 
preservação, a instituições que realizam este trabalho no Brasil ainda são pouco numerosas, considerando a amplitude da costa brasileira. É também de grande importância que os dados existentes possam ser sistematizados em um banco de dados, o qual pudesse ser consultado facilmente por pesquisadores bem como alimentado com novos dados. Uma alternativa é a utilização de ferramentas computacionais de Geoprocessamento, denominada de Sistema de Informação Geográfica (SIG).

O SIG permite armazenar, manipular e tratar grande volume de dados, e também análises complexas, ao integrar dados de diversas fontes e ao criar bancos de dados geográficos de forma rápida e eficaz (CÂMARA et al., 2001). Se o Sistema de Informações Geográficas estiver ligado à internet isto possibilita seu acesso rápido, em tempo real ou quase real (MORAES et al., 2004), o que contribuiu para uma democratização das informações, esta é uma das vantagem deste sistema, a maior cooperação entre os pesquisadores.

Para Lorini et al. (1996) por suas características intrínsecas, o geoprocessamento afigura-se como ferramenta de grande interesse para a biologia da conservação e gestão da biodiversidade, assumindo importante papel na conquista do desafio maior de congregar os resultados científicos e integrá-los aos processos decisórios de planejamento, implantação e monitoramento das políticas de desenvolvimento.

Dentre os trabalhos realizados com o SIG, tendo como objeto de estudo o cetáceo, os de ocorrência e distribuição espacial são importantes, pois, permitem verificar a existência de padrão populacional, que contribuem para a compreensão das causas de morte e tomada de decisão para a conservação destes animais, e, consequentemente do equilíbrio do ambiente marinho.

Um exemplo bem sucedido de SIG-Mamífero Marinho é o Sistema de Monitoramento de Mamíferos Marinhos - SIMMAM, que tem como objetivo coletar e disponibilizar dados sobre avistagem e encalhe de mamíferos marinhos na costa brasileira para a comunidade científica e em geral, disponível na internet no endereço eletrônico http://siaiacad09.univali.br/simmam. 
O SIMMAM permite busca de informações sobre a ocorrência de uma ou mais espécies, envolvendo buscas bibliográficas e contatos com pesquisadores. No sistema a busca é feita com agilidade e segundo Moraes et al. (2004), isto permite a tomada de decisões sobre aspectos ambientais de modo mais rápido e efetivo, diminuindo o grau de incertezas no que se refere aos cetáceos.

O Biomapas é também outro exemplo de banco de dados sobre a ocorrência de animais marinhos na costa brasileira, realizado pelo Petróleo Brasileiro S.A. - Petrobras no site da internet alocado no endereço eletrônico http://www.petrobras.com.br/biomapas/maritimo, que permite conhecer as diversidades de espécies.

Considerando a existência do material tombado sobre cetáceos encontrados nos museus do Paraná e sua importância para o conhecimento de sua ocorrência e distribuição, é o objetivo deste trabalho elaborar um banco de dados utilizando o Sistema de Informação Geográfica, a partir de dados coletados no Museu de Historia Natural Capão da Imbuia e do Museu de Ciências Naturais no Estado do Paraná. Este banco de dados também contribuirá para futuros trabalhos que buscam estabelecer padrão de distribuição de ocorrência de cetáceos na costa paranaense.

\section{ÁREA DE ESTUDO}

O litoral paranaense compreende os municípios de Guaraqueçaba, Antonina, Morretes, Paranaguá, Pontal do Paraná, Matinhos e Guaratuba (Figura 01). Seu território corresponde a 3\% do Estado, limita-se ao norte com o Estado de São Paulo, na vila de Ararapira (S 251ำ44" e $O$ 4801'15") e ao sul com o Estado de Santa Catarina, onde se localiza a barra do rio Saí-Guaçú (S 2558'38" e O 4835'26"), (BIGARELLA, 2001; e LEAL, 2000). Possui litoral estreito que desempenha o papel de uma zona de passagem para o oceano (MAACK, 2002). Possui $105 \mathrm{Km}$ de comprimento e é caracterizada por uma rica fisiografia com forma retilínea, orientação NE/SW, praias dissipativas dominadas por ondas e correntes de deriva litorânea (ANGULO, 1993). 
Figura 01 - Municípios do Litoral Paranaense

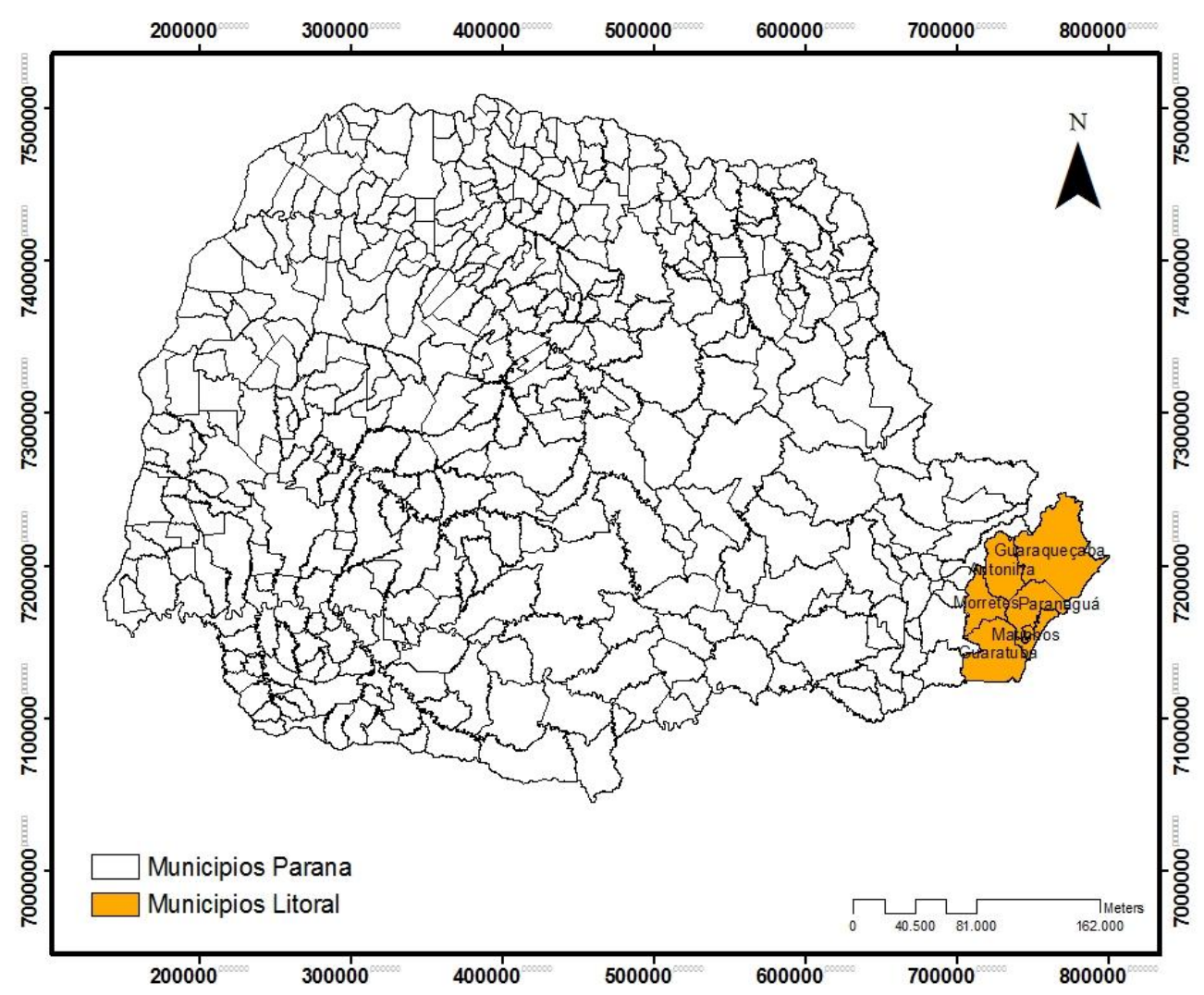

Fonte: ITCG, 2011 - http://www.itcg.pr.gov.br

Org: autor 2011.

A planície litorânea apresenta cerca de 10 a 20 Km de largura, na Baía de Paranaguá é onde atinge o máximo de $50 \mathrm{Km}$, é constituída de formações arenosas, paludais terrestres, manguezais (paludais marinhos) e por terrenos de aluviões terrestres. (BIGARELLA, 2001).

O clima da região é subtropical com chuvas abundantes e verões e invernos bem definidos (MAACK, 2002), favorecendo a formação dos recursos hídricos em praticamente toda a extensão dos remanescentes de Floresta Atlântica. Segundo Anacleto e Almeida (2008), os recursos hídricos no litoral do Paraná vem sofrendo alterações provocadas pela agricultura, onde o uso da água está associado ao uso de agrotóxicos de elevado poder residual, e também pela poluição urbana intensificada pela ausência de consciência da população com o destino inadequado do lixo nos cursos de água existente pelo 
meio urbano, que acabam por poluir estes mananciais, podendo comprometer a reprodução de várias espécies marinhas.

A fauna paranaense é rica em diversidade de biomas e ecossistemas, porém muitas espécies se encontram sob grau de ameaça por motivos como: destruição e redução dos ecossistemas, da caça e pesca predatória, do comércio ilegal de espécimes, da poluição dos ecossistemas terrestres e aquáticos, da introdução de espécies exóticas, da perda de fontes alimentares e do uso indiscriminado de agroquímicos (MIKICH et al.,2004). Os mamíferos aquáticos também são ameaçados pela ação antrópica como, por exemplo: caça comercial, captura acidental em redes de espera, poluição química, degradação de habitat, turismo e tráfego de barcos (COSTA et al., 2005).

Uma das formas de proteger os mamíferos marinhos é a preservação de seu habitat, para tanto é necessário o conhecimento da espécie e de seu entorno. A criação e manutenção de bancos de dados com variáveis, de suas características, comportamento, e a influencia das ações antrópicas, permite a sistematização, o cruzamento de dados e a tomada de decisões.

\section{MATERIAS E MÉTODOS}

O levantamento de dados de materiais tombados foi realizado no Museu de Historia Natural Capão da Imbuia e o Museu de Ciências Naturais da Universidade Federal do Paraná, em Curitiba - PR.

No primeiro museu os dados foram levantados a partir da consulta dos exemplares guardados em armários, os quais são identificados com etiquetas, contendo a espécie, local de captura e nome do coletor. No segundo os dados estavam sistematizados em listagem e foram adquiridos por meio de solicitação encaminhada ao pesquisador MsC. Fernando A. Sedor responsável pelo acervo.

A primeira etapa do desenvolvimento do SIG, que trata da elaboração do banco de dados cartográfico e alfanumérico foi realizada no Software ArcGIS 9.3 a partir do ArcCatalog para criar o Geodatabase ${ }^{3}$. Foi criado

\footnotetext{
${ }^{3}$ Geodatabase: Uma estrutura de banco de dados ou arquivo usado principalmente para armazenar, consultar e manipular dados espaciais (GIS Dictionary ESRI).
} 
Dataset $^{4}$ para melhor organizar os dados e as Feature Classes ${ }^{5}$ que representam os pontos de cada área. Cada Feature Class possui um banco de dados para cada ponto mapeado que gera automaticamente um identificador ID onde podem ser adicionadas as informações necessárias.

A base de dados cartográfica em formato Shapefile ${ }^{6}$ foi importada do site do ITCG <http://www.itcg.pr.gov.br> e as imagens orbitais do LANDSAT 5 TM de 19/11/2010, orbita. 220-077 e LANDSAT 5 TM de 19/11/2010, orbita. 220-078 foram adquiridas no site do INPE <http://www.inpe.br/>. A segunda etapa realizada no ArcMap consistiu na importação das imagens orbitais, a elaboração composição colorida, do mosaico das imagens e da mascara para recorte da área de estudo (Figura 02).

Figura 02 - Mosaico de Imagens Landsat 5 TM da Área de Estudo

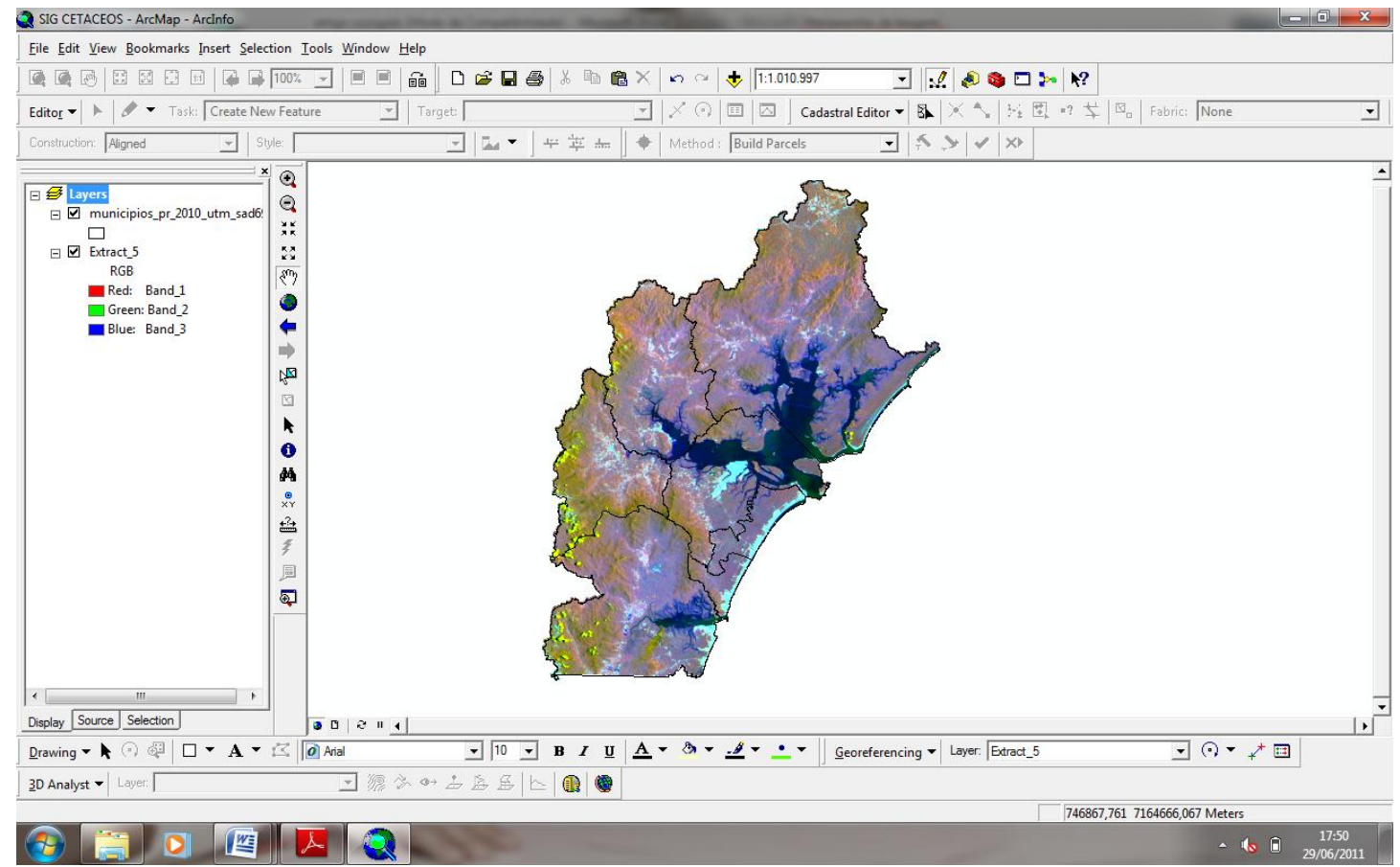

Fonte: autor, 2011.

\footnotetext{
${ }^{4}$ Database: Qualquer coleção de dados relacionados, geralmente agrupados ou armazenados juntos (GIS Dictionary ESRI).

${ }^{5}$ Feature Classes: conjunto de características geográficas com o mesmo tipo de geometria (como ponto, linha ou polígono), os mesmos atributos, e a mesma referência espacial (GIS Dictionary ESRI).

${ }^{6}$ Shapefile: Um vetor de formato de armazenamento de dados para armazenar a localização, a forma e os atributos de características geográficas. Um shapefile é armazenado num conjunto de ficheiros relacionados e que contém uma classe de recursos (GIS Dictionary ESRI).
} 
$\mathrm{Na}$ tabela de atributo referente aos 197 registros dos materiais tombados analisados foram criados cinco campos correspondentes as variáveis: família ou espécie de cetáceos, local de coleta, data de coleta, nome do coletor e o código de tombo, parte desta esta exemplificada na Figura 03. Esta ultima variável consta apenas nos exemplares do Museu de Ciências Naturais da Universidade Federal do Paraná.

Figura 03 - Exemplo parcial da Tabela de Atributos

\begin{tabular}{|c|c|c|c|c|}
\hline FAMÍLIA & LOCAL & DATA & COLETOR & $\begin{array}{l}\text { CÓDIGO } \\
\text { TOMBO }\end{array}$ \\
\hline Delphinidae & $\begin{array}{l}\text { Pontal do Paraná - } \\
\text { Pontal do Sul }\end{array}$ & 1995 & $\begin{array}{l}\text { M. Barletta C. } \\
\text { e Soares }\end{array}$ & $\begin{array}{l}\text { MCN } \\
\text { UFPR } 002\end{array}$ \\
\hline Delphinidae & $\begin{array}{l}\text { Pontal do Paraná - } \\
\text { Praia do Village }\end{array}$ & $\begin{array}{c}25 / \mathrm{VIII} / \\
1989\end{array}$ & R.C. Zanelatto & $\begin{array}{l}\text { MCN } \\
\text { UFPR } 003\end{array}$ \\
\hline Delphinidae & $\begin{array}{l}\text { Pontal do Paraná - } \\
\text { Praia dos Barrancos }\end{array}$ & $\begin{array}{l}07 / \mathrm{VIII} / 1 \\
989\end{array}$ & M.F. Corrêa & $\begin{array}{l}\text { MCN } \\
\text { UFPR } 004\end{array}$ \\
\hline Delphinidae & $\begin{array}{l}\text { Pontal do Paraná - } \\
\text { Praia do Atami }\end{array}$ & $\begin{array}{l}\text { 22/VIII/1 } \\
989\end{array}$ & $\begin{array}{l}\text { R.C. Zanelatto } \\
\text { e M.F. Corrêa }\end{array}$ & $\begin{array}{l}\text { MCN } \\
\text { UFPR } 005\end{array}$ \\
\hline Delp & $\begin{array}{l}\text { Ilha do Superagui - } \\
\text { Praia Deserta }\end{array}$ & $\begin{array}{l}20 / \mathrm{VIII} / 1 \\
996\end{array}$ & Leite, M.R.P. & $\mathrm{MHNCl}$ \\
\hline $\begin{array}{l}\text { Pont } \\
\text { brain }\end{array}$ & $\begin{array}{l}\text { Pontal do Sul - } \\
\text { Paranaguá }\end{array}$ & VII/1991 & Zanellato, R.C. & $\mathrm{MHNCl}$ \\
\hline $\begin{array}{l}\text { Pontoporia } \\
\text { brainillei }\end{array}$ & $\begin{array}{l}\text { Pontal do Sul - } \\
\text { Paranaguá }\end{array}$ & 1998 & $\begin{array}{l}\text { Bedin, S. \& } \\
\text { Moraes, V. }\end{array}$ & $\mathrm{MHNCl}$ \\
\hline $\begin{array}{l}\text { Pontoporia } \\
\text { brainillei }\end{array}$ & Ilha do Mel & 1990 & Moraes, V. & $\mathrm{MHNCl}$ \\
\hline $\begin{array}{l}\text { Pontoporia } \\
\text { brainillei }\end{array}$ & $\begin{array}{l}\text { Ilha do Superagui - } \\
\text { Praia Deserta }\end{array}$ & $\begin{array}{c}24 / 11 / 19 \\
91 \\
\end{array}$ & $\begin{array}{l}\text { Person, V.S. \& } \\
\text { Lorini, M.L. }\end{array}$ & $\mathrm{MHNCl}$ \\
\hline $\begin{array}{l}\text { Sotalia } \\
\text { guianeses }\end{array}$ & Ilha do Mel & $\begin{array}{l}15 / \mathrm{V} / 11 \\
95\end{array}$ & Leite, M.R.P. & $\mathrm{MHNCl}$ \\
\hline
\end{tabular}

Fonte: Levantamento de dados - 2010.

Os 197 registros de ocorrências de cetáceos com seus atributos foram plotados utilizando o Feature Class (Figuras 04 e 05).

A identificação do local de coleta foi nomeada por municípios ou pelo nome da ilha e/ou da praia. Em alguns dos registros estavam identificados apenas com o nome do município ou da ilha, quando isso ocorreu o registro foi plotado no centróide do polígono. Esta generalização quanto à localização do material tombado, não permite uma precisão na plotagem dos pontos, como 
por exemplo, em coordenadas geográficas, o que trariam muito benefícios para estudos aprofundados.

Figura 04 - Localização das Ocorrências de Materiais Tombados na Costa Paranaense

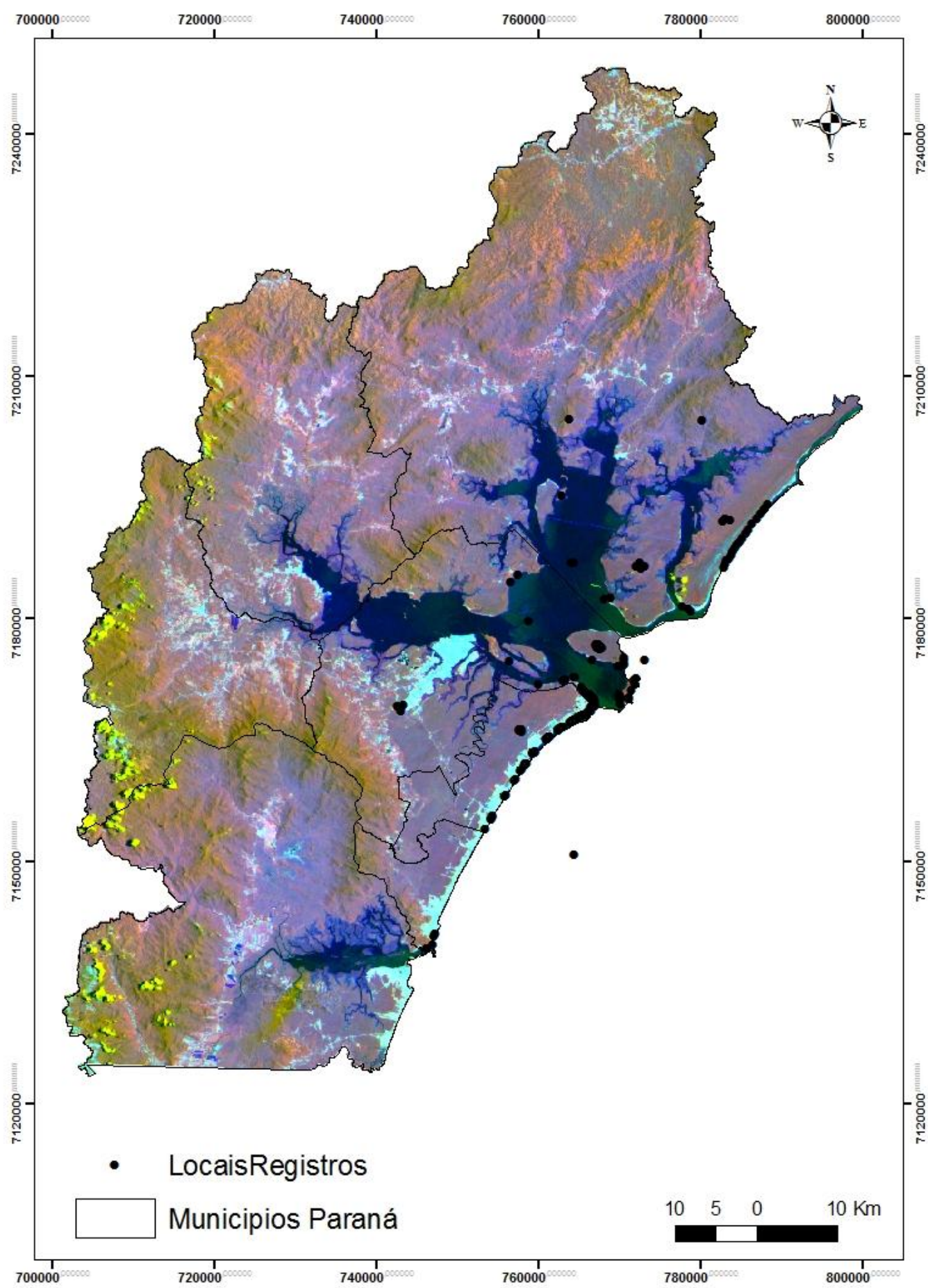

Fonte: autor, 2011. 
Figura 05 - Localização das Ocorrências de Materiais Tombados, em destaque o Município de Pontal do Paraná.

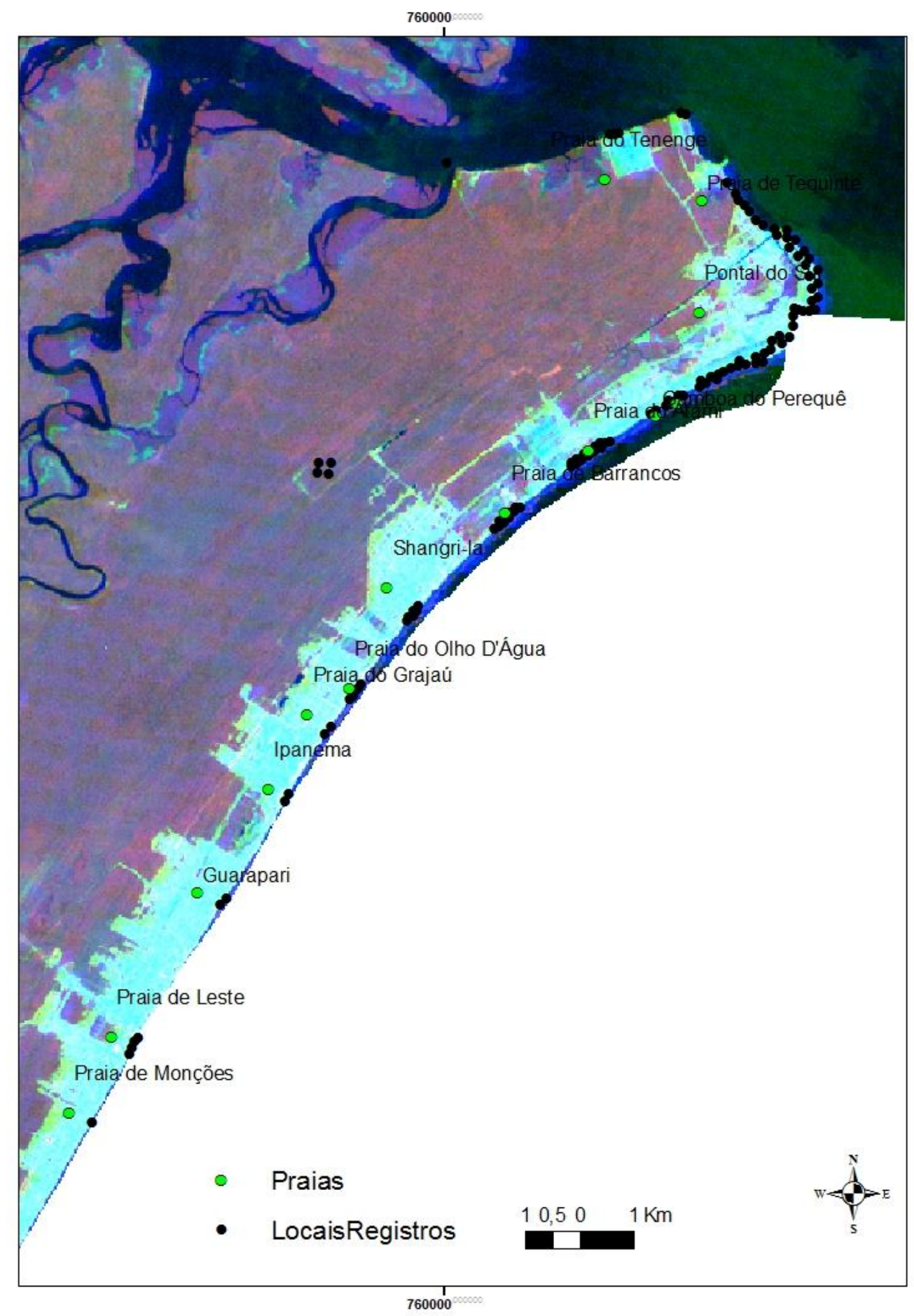

Fonte: autor, 2011 
Cada Feature Class possui um banco de dados, onde cada ponto mapeado gera automaticamente um ID. É possível acessar a tabela de atributos, com a ferramenta Identify (Figura 06).

Figura 06 - Ponto Mapeado e Respectiva Tabela de Atributos

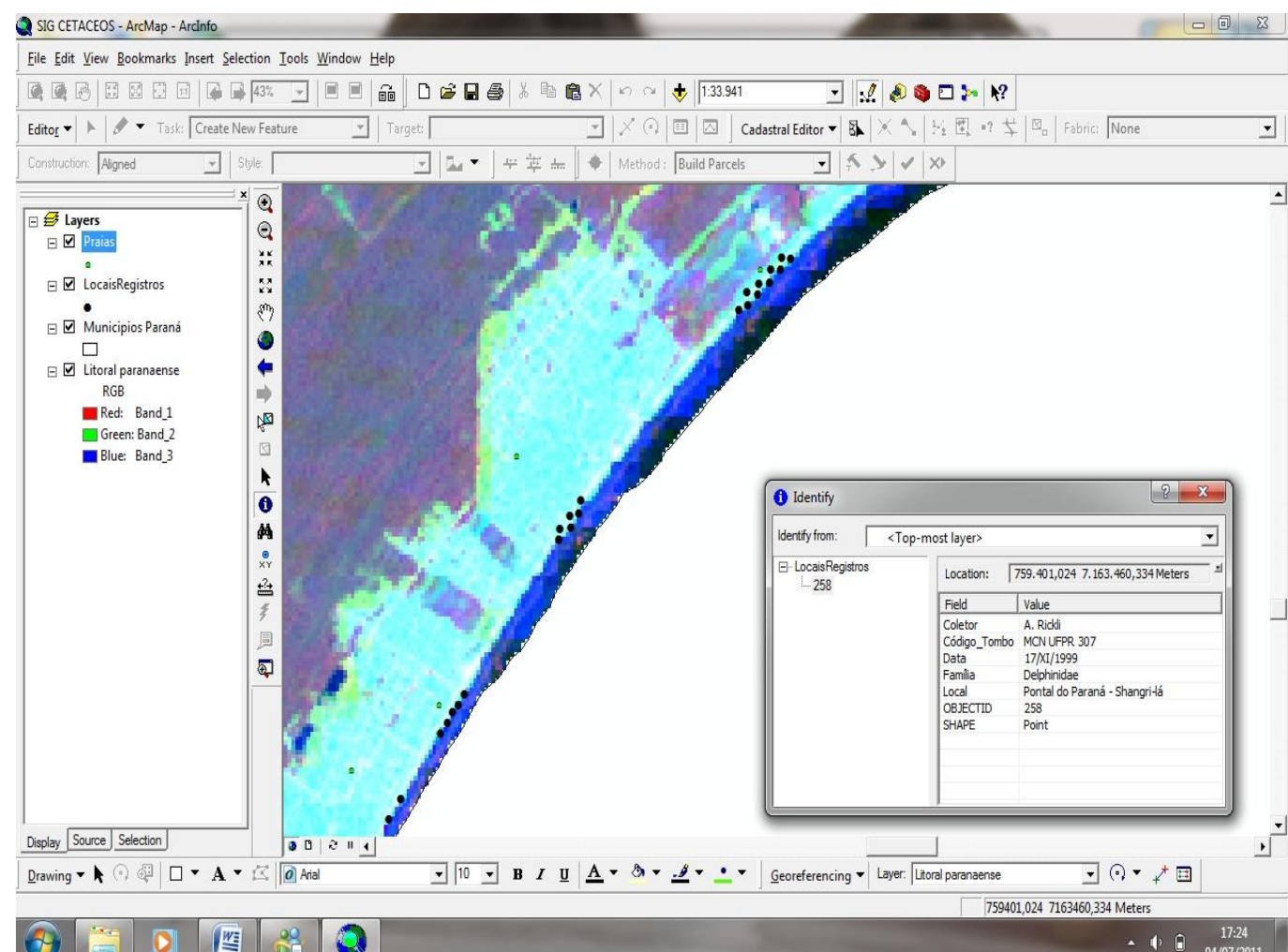

Fonte: autor, 2011

O sistema de Geodatabase suporta todos os tipos de dados que podem ser utilizados pelo ArcG/S, tais como: características geográficas, atributos das tabelas, superfície e modelagem de dados, inquéritos e medições além das imagens de satélite e aéreas. Ele não define apenas o modo que os dados podem ser armazenados como também propõe ao usuário como estes dados podem ser acessados e geridos. Além disso, neste mesmo sistema é possível verificar a modelagem de relações espaciais entre os dados e as validações dos dados (como os subtipos e domínios). O usuário pode, através do Geodatabase, conservar os dados em um único local de forma organizada e sistêmica, manter a integridade dos dados e integrá-los com outras bases de dados além de ser personalizado pelo usuário (POLIDORO \& FERNANDES 
BARROS, 2011). Isto permite que outras variáveis sejam introduzidas, ampliando e/ou modificando o banco de dados.

\section{BANCO DE DADOS-CETÁCEOS PARANÁ}

Os dados de materiais tombados referentes ao período de 1981 a 2005 foram agrupados segundo as localidades e totalizaram 199 ocorrências. Duas ocorrências não foram mapeadas, pois em uma delas não havia a espécie identificada e em outra não estava identificado o local de ocorrência.

A subordem Odontoceti, cetáceos com dentes, é composta por varias famílias. Do material tombado analisado foram identificadas apenas quatro famílias, a Delphinidae com 184 registros, seguido de Pontoporiidae com 11 e Physiteridae e Ziphidae apenas com um registro.

O município que apresentou maior ocorrência foi Pontal do Paraná com 113 registros (Figura 07). Outras localidades com significativas ocorrências foram Ilha do Mel com 35 e Ilha do Superagui com 24. No litoral de Pontal do Paraná foram identificadas 22 localidades (Tabela 01), com maior concentração na praia de Pontal do Sul com 49.

O ano com maior incidência de animais foi 1992 (Figura 08), e os meses foram julho e agosto respectivamente (Figura 09).

Figura 07: Local de registro de materiais tombados

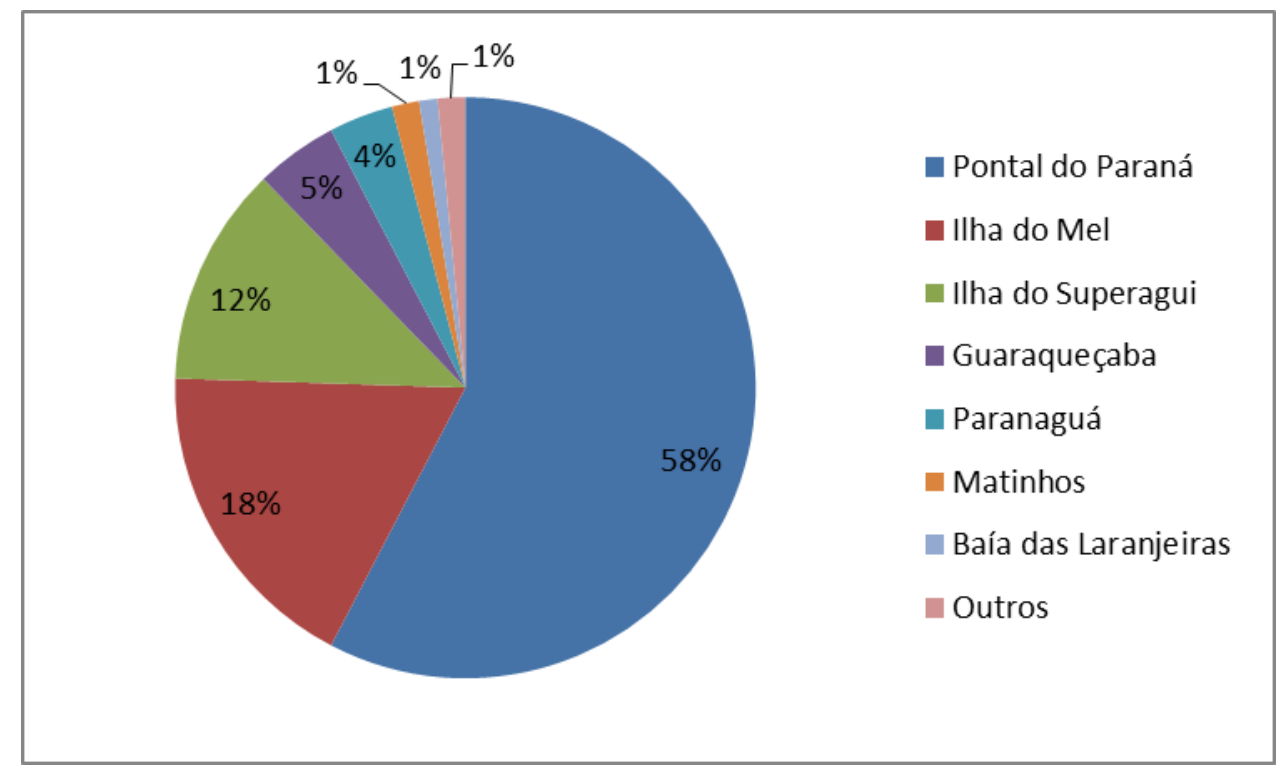

Fonte: Levantamento de dados - 2010. 
Tabela 01: Local de Registro de Materiais Tombados

\begin{tabular}{|c|c|}
\hline Pontal do Paraná & Registros \\
\hline Pontal do Sul & 49 \\
\hline Praia do Atami & 13 \\
\hline Praia de Barrancos & 10 \\
\hline Praia do Village & 7 \\
\hline Shangui-lá & 6 \\
\hline Praia do Olho D’Água & 5 \\
\hline Praia de Leste & 4 \\
\hline Balbeário Grajaú & 2 \\
\hline Gamboa do Perequê & 2 \\
\hline Ipanema & 2 \\
\hline Ponta do Poço & 2 \\
\hline Pontal do Paraná & 1 \\
\hline Balneário Santa Terezinha & 1 \\
\hline Guarapari & 1 \\
\hline Praia de Massarapuã & 1 \\
\hline Praia de Monções & 1 \\
\hline Praia de Tequinte & 1 \\
\hline Praia do Tenenge & 1 \\
\hline Prainha próximo à Tenegue & 1 \\
\hline Praia de Florida & 1 \\
\hline Próximo à praia de Tenengue & 1 \\
\hline Próximo à Transturmar & 1 \\
\hline
\end{tabular}

Fonte: Levantamento de dados - 2010, 
Figura 08: Numero de Registro de Material Tombado - entre 1985 a 2005

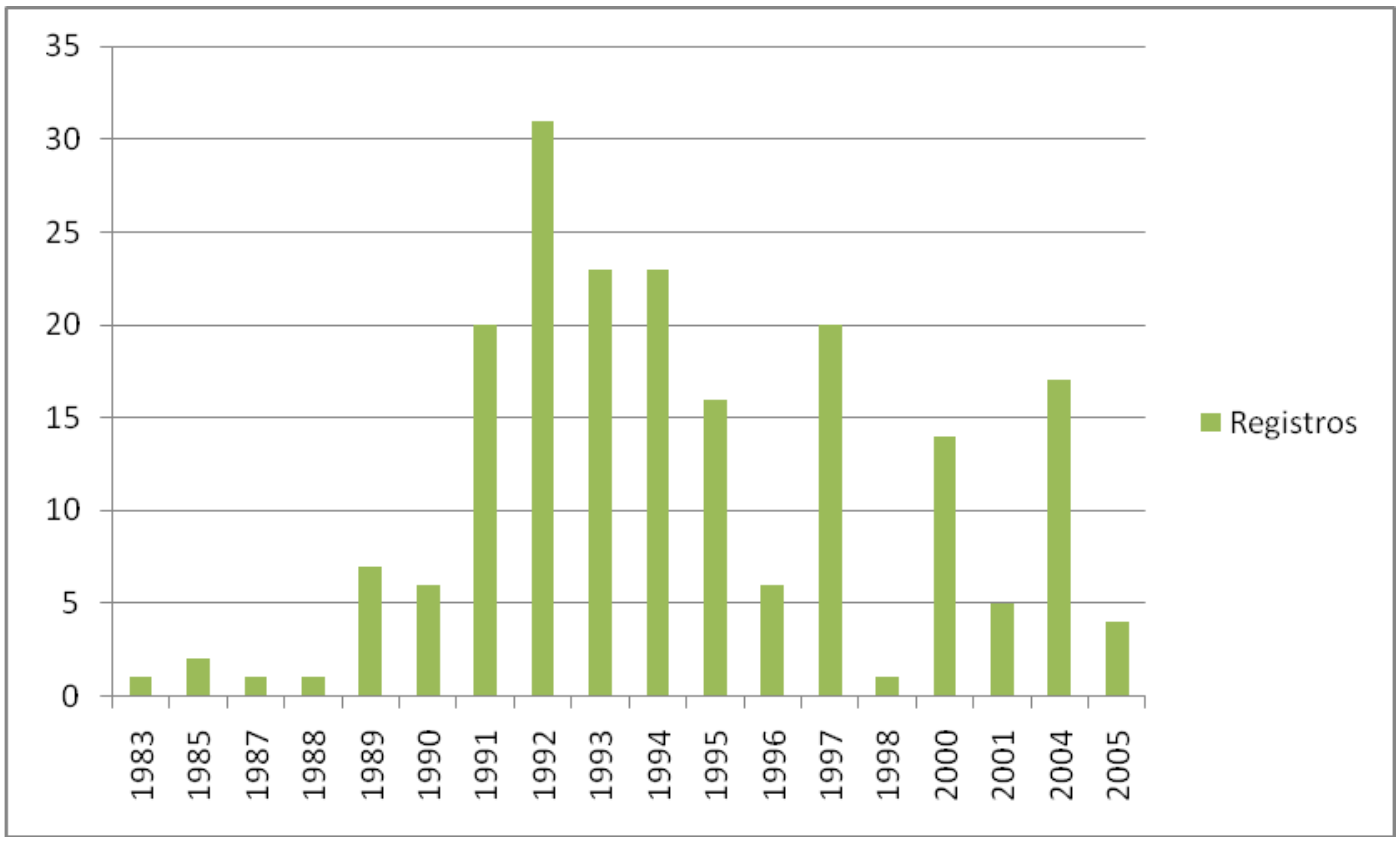

Fonte: Levantamento de dados - 2010.

Figura 09: Numero de registro de materiais tombados - por mês nos anos 1985 a 2005

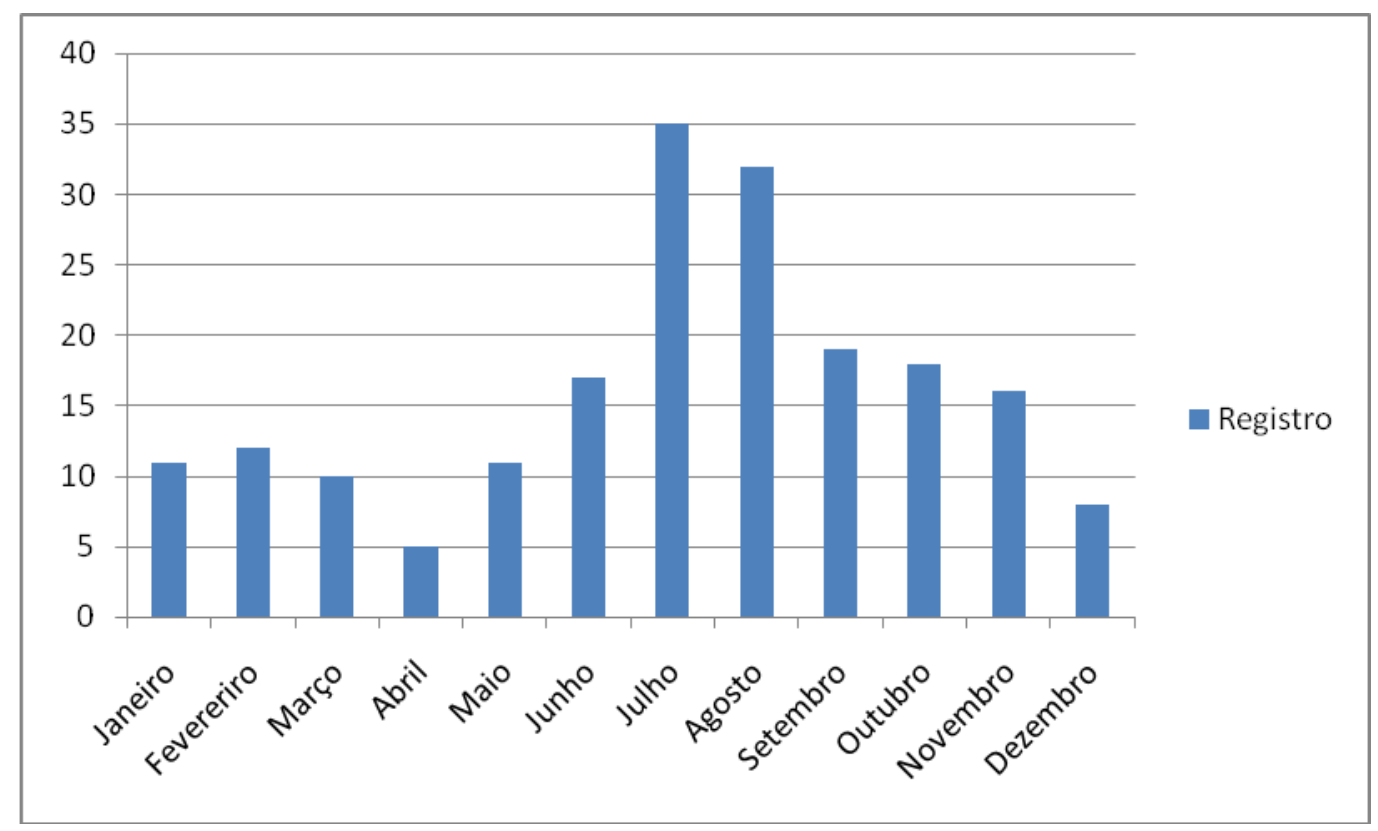

Fonte: Levantamento de dados - 2010. 
A família Delphinidae apresenta dentes cônicos e curvos, possuem grande variação em tamanho e contribuem com pelo menos 36 espécies, entre elas orca (Orcinus orca), golfinho rotador (Stenella longirostris), boto-cinza (Sotalia guianensis) e golfinho nariz de garrafa (Tursiops truncatus). Vários estudos indicam que a espécie que habita a região é a Sotalia guianensis, boto-cinza.

Sotalia guianensis, espécie citada no Plano de Ação para Mamíferos Aquáticos do Brasil como uma espécie vulnerável (IBAMA, 2001); ocorre frequentemente em águas costeiras como baías e estuários, sua distribuição é provavelmente contínua desde Honduras até Santa Catarina, Sul do Brasil (CREMER et al., 2009); essa espécie está sob um risco alto de extinção na natureza e tem ocorrência registrada ao longo de todo o ano no estado do Paraná (FILLA 1999, 2004; MONTEIRO-FILHO et al, 1999; BONIN, 2001; e DOMIT, 2006) e utiliza a região para alimentação (OLIVEIRA 2003; e DOMIT, 2006), reprodução (ROSAS \& MONTEIRO-FILHO, 2002) e cuidado dos infantes (RAUTENBERG, 1999; e DOMIT, 2006).

Estudos realizados por Rosas (2000); Domit et al. (2008); Londoño et al. (2008), apontam esta espécie como sendo as com maiores taxas de mortalidade (animais encalhados em praia). A taxonomia é bastante controversa, pois alguns autores consideram duas espécies, S. fluviatilis para população amazônica e $S$. guianensis para população marinha (MONTEIRO FILHO; MONTEIRO \& REIS, 1999). Outros consideram uma única espécie, com duas subespécies, S. f. fluviatilis e S. f. brasiliensis (RICE, 1998), enquanto que vários autores consideram apenas uma espécie com duas populações ou ecótipos: um marinho e outro fluvial (BOROBIA, 1989).

A família Pontoporiidae é representada pelas toninhas, Pontoporia blainvillei, são de pequeno porte e usam águas costeiras que vão do Estado do Espírito Santo ao Norte da Argentina (SIMÕES-LOPES, 2006). É a espécie mais ameaçada no Brasil e citada no Plano de Ação para Mamíferos Aquáticos do Brasil como EM - em perigo (IBAMA, 2001).

No Paraná sua ocorrência é ao longo de todo o ano e desde 2007 há registros na área interna do Complexo Estuarino de Paranaguá (SANTOS et 
al., 2010). Segundo Domit et al. (2008) e Londoño et al. (2008) a toninha apresenta a segunda maior taxa de mortalidade (animais encalhados em praia).

A Physiteridae apresenta enorme cabeça, sopro de vapor da respiração para frente e o dimorfismo sexual em tamanho é surpreendente entre os cetáceos, principalmente no grande cachalote, Physeter macrocephalus), espécie é cosmopolita (SIMÕES-LOPES, 2005).

A Ziphidae é representada pelas baleias-de-bico, pois possuem focinho alongado semelhante a um bico, são amplamente distribuídas. A Baleiabicuda-de-Cuvier, Ziphius cavirostris, é um representante dessa família, espécie migratória (SIMÕES-LOPES, 2005).

O numero de ocorrência de Delphinidae é muito superior às demais famílias identificadas. Diversos estudos realizados na área indicam a predominância de Sotalia guianensis pertencente aos delfinideos, porem, esta constatação é prejudicada pela ausência da identificação da espécie no registro de tombamento, o que enfatiza a importância da variável espécie na catalogação do material tombado.

A distribuição espacial apresenta-se concentrada no município Pontal do Paraná, com maior ocorrência em Pontal do Sul, o que pode ser explicado pelo fato da área ser objeto de inúmeras pesquisas, principalmente pelo Centro de Estudos do Mar - UFPR. Esta espacialização não pode ser considerada como padrão de ocorrência para o Paraná, já que inúmeros exemplares são perdidos pela falta da coleta.

Com ampliação da coleta de material tombado e sua sistematização em um banco de dados em ambientes SIG, que permita acesso para consulta e atualização por diferentes instituições e/ou pesquisadores, certamente irá contribuir para o desenvolvimento e avanço de inúmeras pesquisas, e, para o monitoramento desses mamíferos aquáticos.

\section{CONSIDERAÇÕES}

Os cetáceos são importantes na cadeia alimentar e consequentemente no equilíbrio do ecossistema aquático. O conhecimento da sua população, do 
seu habitat, do seu comportamento, permite sua preservação por meio de políticas de monitoramento e preservação.

A localização e frequência das ocorrências em conjunto com outras variáveis do ambiente costeiro (clima, condições ambientais, qualidade da água, corrente marítima) podem permitir estabelecer diretrizes de monitoramento, controle e preservação destas espécies. Por esse motivo é importante que os dados de avistagem, captura acidental, encalhe e de materiais tombados tenham registros com a localização precisa, e, se possível em coordenadas geográficas.

A elaboração e manutenção de banco de dados digitais das ocorrências de encalhe, avistagem e captura acidental desta espécie permite por um lado à democratização do acesso ao dado e seu acúmulo e por a definição de padrão de ocorrência e consequentemente suporte a tomada de decisão.

Estabelecer padrão de ocorrência contribui para o conhecimento da vida desses mamíferos que aliando a outras variáveis de caráter ambiental permite uma melhor compreensão sobre esta espécie e sua interação com o meio que vive.

Esse trabalho buscou sistematizar dados sobre a ocorrência de cetáceos no litoral paranaense a partir de dados tombados de 1981 a 2005, estes nos permite apenas estabelecer parcialmente um perfil sobre esta ocorrência, pois as amostras são em número reduzido além da peculiaridade deste tipo de material.

Ressalta-se a importante da coleta e da sistematização de dados de materiais tombados, com objetivo de conhecer as espécies e seu padrão de distribuição especial, com a finalidade de preservação da vida desses cetáceos.

\section{REFERÊNCIAS BIBLIOGRÁFICAS}

ANACLETO Adilson \& ALMEIDA Iracema Batista de. A educação como gestão estratégica para preservação dos recursos hídricos no litoral do Paraná. In: CONGRESSO INTERNACIONAL DE ADMINISTRAÇÃO - GESTÃO NA ERA 
DO CONHECIMENTO, 2008, Ponta Grossa: Editora, Universidade Ponta Grossa, 08 a 12 de Setembro de 2008, 8p.

ANGULO, R. T. 1993. A Ocupação Urbana do Litoral Paranaense e as Variações da Linha de Costa. Boletim Paranaense de Geociências, Curitiba, 41: 73-81.

BIGARELLA, João José. Contribuição ao Estudo da Planície Litorânea do Estado do Paraná. Brazilian Archives of Biology and Technology. Curitiba, vol jubilee, p.65-110, 2001.

BONIN, Carolina Aimoré. Utilização de habitat pelo Boto-cinza, Sotalia fluviatilis guianensis (Cetacea, DELPHINIDAE), na porção norte do Complexo estuarino da baía de Paranaguá, PR. 2001. Dissertação (Mestrado em Zoologia), Departamento de Zoologia, Universidade Federal do Paraná, Curitiba.

BOROBIA, Monica. Distribution and morphometrics of South American dolphins Sotalia. 1989. Dissertação (Master of Science) Department of Renewable Resources, McGill University, Montreal.

CÂMARA, Gilberto; DAVIS, Clodoveu; MONTEIRO, Antônio Miguel. Introdução à Ciência da Geoinformação. São José dos Campos: INPE, 2001.

COSTA, Leonora P.; LEITE, Yuri Luiz R.; MENDES, Sérgio L. \& DITCHIFIELD, Albert David. Conservação de mamíferos no Brasil. Megadiversidadev.1, $n^{\circ}$ 1, p. 103-122, 2005.

CREMER, Marta Jussara; SIMÕES-LOPES Paulo César \& PIRES José Salatiel Rodrigues. Occupation Pattern of a Harbor Inlet by the Estuarine Dolphin, Sotalia guianensis (P. J. Van Bénéden, 1864) (Cetacea, Delphinidae). Brazilian Archives of Biology and Technology, v.52, n.3, p. 765-774, 2009.

DOMIT, Camila; ROSA, Liana; SASAKI, Glaucia; LONDOÑO, Maria Camila Rosso \& LOPES, Ana Rita Dos Santos. Progress Report on cetacean research, March 2008 to March 2009, with statistical data for calendar year 2008. International Whaling Commission. 6p. 2008.

FILLA, Gislaine Fatima. Estimativa da densidade populacional e estrutura de agrupamento do boto-cinza Sotalia guianensis (Cetacea: Delphinidae) na baía de Guaratuba e na porção norte do Complexo Estuarino da Baía de Paranaguá, PR. 2004. Dissertação (Mestrado em Zoologia), Departamento de Zoologia, Universidade Federal do Paraná, Curitiba.

FILLA, Gislaine Fatima. Estimativa da densidade populacional de filhotes e estrutura de grupo do boto-cinza Sotalia guianensis (Cetacea: Delphinidae) na Baía de Guaraqueçaba e na llha das Peças, Litoral do 
Estado do Paraná. 1999. Monografia de Bacharelado, Departamento de Zoologia, Universidade Federal do Paraná, Curitiba, PR.

GIS Dictionary da ESRI. Disponível em: http://support.esri.com/en/knowledgebase/GISDictionary/ . Acessado em: 04 de setembro de 2012.

HETZEL Bia \& LODI Liliane. Baleias, botos e golfinhos: guia de identificação para o Brasil. Rio de Janeiro: Editora Nova Fronteira, 1993.

IBAMA. Instituto Brasileiro de meio ambiente de recursos naturais renováveis. Mamíferos Aquáticos do Brasil: Plano de Ação, versão II. Brasília, Edições IBAMA, 2001.

INPE. Disponível em: http://www.inpe.br/. Acessado em: 31 de março de 2011.

ITCG. Divisão Político-Administrativa do Paraná - 2010. Disponível em: http://www.itcg.pr.gov.br. Acessado em: 31 de março de 2011.

LORINI, Maria Lucia; PERSSON Vanessa Guerra \& XAVIER DA SILVA Jorge. Geoprocessamento aplicado à conservação de espécies ameaçadas de extinção: o projeto mico-leao-da cara-preta. In: 1a SEMANA ESTADUAL DE GEOPROCESSAMENTO, 1996, Rio de Janeiro, p. 147-158.

MAACK, Reinhard. Geografia Física do Estado do Paraná. Curitiba: Imprensa Oficial, 2002, 2 $2^{\mathrm{a}}$ edição. $1^{\mathrm{a}}$ edição em 1968. 350 p.

MEDEIROS, Priscila Izabel Alves Pereira de. Encalhe de Cetáceos ocorridos no período se 1984 a 2005 no litoral do Rio Grande do Norte, Brasil. 2006. Dissertação de Mestrado em Bioecologia Aquática. Departamento de Oceanografia e Limnologia, Universidade Federal do Rio Grande do Norte Centro de Biociências, Natal.

MIKICH, Sandra, BÉRNILS Renato Silveira e PIZZI Paulo Aparecido. Livro Vermelho da Fauna Ameaçada no Estado do Paraná. Editora, Mater Natura, 2004. Disponível em: http://www.maternatura.org.br. Acesso em: 27 de agosto de 2012.

MONTEIRO-FILHO, Emygdio Leite de Araujo; MONTEIRO L. R. \& REIS S. F. Geometric analysis of tridimensional skull shapa in Sotalia: discrimination between freshwater and marine dolphins. In: BIENNIAL CONFERENCE ON THE BIOLOGY OF MARINE MAMMALS, 13, 1999, Maui: Proceeding...Maui, 28 de novembro-dezembro 3,1999. p129.

MONTEIRO-FILHO, Emygdio Leite de Araujo; BONIN, Carolina Aimoré \& RAUTENBERG, Marina. Interações interespecíficas dos mamíferos marinhos na região da Baía de Guaratuba, litoral sul do Estado do Paraná. Biotemas, v.12, cap.1, p.119-132, 1999. 
MORAES, Cristiane Gonçalves de. Sistema de monitoramento de mamíferos marinhos - SIMMAM: uma ferramenta para o estudo de avistagem e encalhes na Costa Brasileira. 2005. Dissertação (Mestrado em Ciências e Tecnologia Ambiental), Centro de Ciências Tecnológicas da Terra e do Mar, Universidade do Vale do Itajaí, Itajaí.

MORAES, Cristiane Gonçalves de; André Silva BARRETO \& R. M. SPERB. Sistema de Informação Geográfica para dados de encalhes e avistagens de Cetáceos. In: IV CONGRESSO BRASILEIRO DE COMPUTAÇÃO-CBCOMP, 2004, Itajaí, Anais do IV CBComp. Itajaí, SC: Univali, CTTMar, 2004. p. 820822.

OLIVEIRA, Márcia Regina de. Ecologia alimentar de Sotalia guianensis e Pontoporia brainvillei (Cetacea, Delphinidae e Pontoporiidae) no litoral do estado de São Paulo e litoral do estado do Paraná. 2003. Dissertação (Mestrado em Zoologia), Departamento de Zoologia, Universidade Federal do Paraná, Curitiba.

POLIDORO, Mauricio \& Mirian Vizintim FERNANDES-BARROS. Proposta metodológica de desenvolvimento de Sistema de Informações Geográficas em ambiente web (WebGIS) aplicado ao turismo. Barcelona: Ar@cne, 2011. Disponível em: http://www.ub.edu/geocrit/aracne/aracne-133.htm. Acesso em: 1 de maio de 2010.

RAUTENBERG, Marina. Cuidados parentais de Sotalia fluviatilis guianensis (Cetacea, Delphinidae) na região do complexo estuarino lagunar Cananéia-Paranaguá. 1999. Dissertação (Mestrado em Zoologia), Departamento de Zoologia, Universidade Federal do Paraná, Curitiba.

REYNOLDS John E. \& ROMMEL Sentiel A. Biology of Marine Mammals. Washington: Smithsoniam Instituition Press, 1999.

RICE, Dale W. Marine mammals of the world. Systematic and distribution. The Society of Marine Mammalogy, 1998.

ROSAS, Fernando C. Weber \& MONTEIRO-FILHO Emygdio Leite de Araujo. Reproduction of the estuarine dolphin (Sotalia guianensis) on the coast of Paraná, Southern Brazil. Journal of Mammalogy, v.83, cap.2, p.507-515, 2002.

ROSAS, Fernando C. Weber. Biologia reprodutiva de Sotalia guianensis e Pontoporia blainvillei no litoral sul do estado de São Paulo e litoral do estado do Paraná. 2000. Tese de Doutorado, Departamento de Zoologia,Universidade Federal do Paraná.

LONDOÑO, Maria Camila Rosso; DOMIT Camila; SASAKI Glaucia; ROSA Liana; GUEBERT F. M.; LÓPEZ E. A. \& MONTEIRO-FILHO Emygdio Leite de Araujo. Encalhe de Cetáceo no litoral do estado do Paraná, sul do Brasil. In: XIII REUNIÓN DE TRABAJO DE ESPECIALISTAS EN MAMÍFEROS 
ACUÁTICOS DE AMERICA DEL SUR - 7ํ CONGRESO SOLAMAC, 2008, Montevideo. "Estatus de los mamiferos acuáticos en Latinoamérica". Montevideo, 2008.

SANTOS, M. C. O; J. E. F. OSHIMA; E. S. PACIFICO \& E. SILVA. Group size and composition of Guiana dolphins (Sotalia guianensis) (Van Bénèden, 1864) in Paranaguá Estuarine Complex, Brasil. Braz. J. Biol., v. 70, $n^{\circ}$. 1, p. 111120, 2010.

SIMMAM. Sistema de Monitoramento de Mamíferos Marinhos. Disponível em: $<$ www.siaiacad09.univali.br/simmam/index.php $>$. Acessado em: 6 de julho de 2010.

SIMÕES-LOPES, Paulo César. O Luar do Delfim. Joinville: Editora, Letradágua. 2005. 304p.

Recebido em 20/03/2012. Aceito em 08/09/2012. 